\title{
Computer aided determination of a Fibonacci group
}

\section{George Havas}

The Fibonacci group $F(2,7)$ has been known to be cyclic of order 29 for about five years. This was first estab].ished by computer coset enumerations which exhibit only the result, without supporting proofs. The working in a coset enumeration actually contains proofs of many relations that hold in the group. A hand proof that $F(2,7)$ is cyclic of order 29 , based on the working in computer coset enumerations, is presented here.

\section{Introduction}

Interest in Fibonacci groups was aroused by Conway [3]. The groups have been studied in detail by Johnson, Wamsley, and Wright [4]. The Fibonacci groups $F(2, n)$ may be presented

$$
\begin{aligned}
& F(2, n)=\left\langle x_{1}, x_{2}, \ldots, x_{n}\right| x_{1} x_{2}=x_{3}, \ldots, x_{n-2} x_{n-1}=x_{n}, x_{n-1} x_{n}=x_{1}, \\
& x_{n} x_{1}=x_{2}>\text {. }
\end{aligned}
$$

A first question about these groups is whether they are finite or not; this has been resolved (see Brunner [1]) for all bar $F(2,9)$. $(F(2,9)$ is still unknown although it is known to have a largest nilpotent quotient of order 152 .)

The groups $F(2, n)$ which are known to be finite have all been identified. In spite of information to the contrary (in [4] and [7]), $F(2,7)$ is cyclic of order 29 . This has been established by coset

Receivea 15 June 1976. 
enumeration on computer, but till now no hand proof has been available.

Given a coset enumeration which shows that a subgroup is of finite index in a certain group, Leech [5] describes a technique for expressing a word in the generators of the group which is in the subgroup as a word in the generators of the subgroup. He amplifies this method in [6] for the proof of relations which hold in the group and describes a computer implementation. This method is the basis of the proof that $F(2,7)$ is cyclic of order 29 presented here.

\section{Notation}

For brevity $x_{i}$ is sometimes denoted by $i$ and $x_{i}^{-1}$ by $-i$. The group identity is denoted by $e$. Thus $F(2,7)$ may be presented

$$
\text { (1) } \begin{aligned}
F(2,7)=\langle 1,2,3,4,5,6,7| 12-3 & =23-4=34-5 \\
= & 45-6=56-7=67-1=71-2=e) .
\end{aligned}
$$

The terminology and notation used in discussion and description of coset enumerations follows [2].

\section{Computer considerations}

It is moderately easy to establish that $F(2,7)$ is cyclic of order 29 by coset enumeration. The easiest way is to observe that the quotient of $F(2,7)$ obtained by abelianizing the presentation is cyclic of order 29 , so it suffices to show that any one of the $x_{i}$ alone generates $F(2,7)$. Each of the coset enumerations $F(2,7) \mid\left\langle x_{i}\right\rangle$, though pathological, is easy enough by machine and gives index 1 as required.

In the context of the Leech method of relation proof from coset enumeration working the most important consideration is the avoidance of coincidences (hence the minimization of total cosets defined). It follows that the Felsch method of coset enumeration is preferred for machine approach to this problem. A Canberra implementation, developed by W.A. Alford, is used for all coset enumerations mentioned here, and is the basis of a computer implementation of Leech's procedure used for relation proof.

The Feisch method yields the following statistics for coset enumerations in $F(2,7)$ presented as in (1). 


$\begin{array}{ccc}\text { Subgroup } & \text { Index } & \text { Total cosets defined } \\ \left\langle x_{1}\right\rangle & 1 & 404 \\ \left\langle x_{2}\right\rangle & 1 & 615 \\ \left\langle x_{3}\right\rangle & 1 & 332 \\ \left\langle x_{4}\right\rangle & 1 & 742 \\ \left\langle x_{5}\right\rangle & 1 & 336 \\ \left\langle x_{6}\right\rangle & 1 & 327 \\ \left\langle x_{7}\right\rangle & 1 & 366 \\ \langle e\rangle & 29 & >33000\end{array}$

It is easy to rewrite the presentation for $F(2,7)$ in terms of two generators by the use of Tietze transformations. A two generator presentation for $F(2,7)$ is:

(2) $\quad F(2,7)=\langle 1,2 \mid 1121-2-21-2-21-2=12-12-122-12-122-122=e\rangle$.

This presentation is much harder for coset enumeration over ( 1 ) and $\langle 2\rangle$ as the following statistics indicate. (Other easily obtained two generator presentations are better, but all I have tried give significantly worse enumerations than the best of the 7 generator enumerations.)

$\begin{array}{ccc}\text { Subgroup } & \text { Index } & \text { Total cosets defined } \\ \langle 1\rangle & 1 & 1143 \\ \langle 2\rangle & 1 & 3103 \\ \langle e\rangle & 29 & 75498\end{array}$

Using Leech's method it is theoretically possible to obtain a proof that $F(2,7)$ is cyclic of order 29 from any one of these enumerations. However, as already mentioned, it is desirable to minimize coincidences so the enumeration $F(2,7) \mid\left\langle x_{6}\right\rangle$ looks the most attractive of the above enumerations. As a starting point for application of Leech's method of relation proof I am unable to find any substantially better enumeration which yields the desired result.

Unfortunately direct appiication of Leech's method to this enumeration, $F(2,7)(\langle 6)$, would lead to a tremendously long proof. The problem arises from the large number of coincidences involved. By studying the coset enumeration I have been able to find a lemma which leads to a 
better coset enumeration (fewer coincidences). The lemma is reasonably easily proved using Leech's method.

The lemma arises this way. In the enumeration $F(2,7) \mid\langle 6\rangle$ the first coset coincidence (which precipitates the total collapse) implies $-1-34464 \epsilon\langle 6\rangle$. Let $h_{1}=-1-34464$. Promisingly, the enumeration $F(2,7) \mid\left(6, h_{l}\right\rangle$ involves the definition of only 177 cosets, as against 327 for $F(2,7) \mid\langle 6\rangle$.

The proof, by Leech's method, of the relation corresponding to the first coincidence involves only 29 of the 327 cosets defined, and of course no other coincidence. The proof of Lemma 1 presented below is essentially that given by Leech's method, abbreviated by the combination of the 110 separate substitutions or reductions given by the method.

Still 177 cosets and coincidences would yield a terribly long proof of the desired result, so I looked for another lemma. In the performance of $F(2,7) \mid\left(6, h_{1}\right)$ the first coincidence, after the definition of 102 cosets, leads to a three coset collapse, and is not of great value. However the fourth coincidence precipitates the total collapse and provides a valuable lema, $-4-6-232464 \in\left\langle 6, h_{1}\right\rangle$. Let $h_{2}=-4-6-232464$. The enumeration $F(2,7) \mid\left(6, h_{1}, h_{2}\right)$ involves the definition of 36 cosets.

Again proof of the corresponding relation is easy enough. The proof involves 46 of the 177 cosets. (The subproof that

$$
-16^{2} h_{1}^{-1} 6^{-2} h_{1} 6^{2}-1-344-2=63
$$

involves the third coincidence, the only time one of the first three coincidences is used.)

The processing of the 36 cosets and coincidences by Leech's method is still too long, so I found one further lemma analogously (from the first coincidence, which precipitates the total collapse) namely $-47-34464 \in\left\langle 6, h_{1}, h_{2}\right\rangle$. Let $h_{3}=-47-34464$. The proof of the corresponding relation involves 13 cosets.

The enumeration $F(2,7) \mid\left(6, h_{1}, h_{2}, h_{3}\right)$ needs 8 cosets. From the 
deduction that generator 5 applied to coset 1 yields coset 1 , the method gives a proof that $5 \in\left(6, h_{1}, h_{2}, h_{3}\right)$, involving all 8 cosets.

The method used here for lemma production looks nicely mechanical. Find the first important coincidence and add the corresponding subgroup generator to the initial subgroup to get an easier enumeration.

Unfortunately it does not always work with the enumeration method used.

The index 2 enumeration $F(2,9)+(1,2)^{2} \mid\langle 1,-23,2-9\rangle$ requires the definition of 39 cosets. The first coincidence, which precipitates the collapse to two cosets, implies that $4-7-25 \in\langle 1,-23,2-9\rangle$. However the enumeration $F(2,9)+(1,2)^{2} \mid\langle 1,-23,2-9,4-7-25)$ requires the definition of 44 cosets.

Note that the relations corresponding to the coincidences and the deduction actually give expressions for $h_{1}, h_{2}, h_{3}$, and 5 in terms of the corresponding subgroup generators, which is rather more than is required for the proof that $F(2,7)$ is cyclic.

\section{Theorem and proof}

THEOREM. The group $F(2,7)$ is cyclic of order 29 .

Proof. The proof follows directly from four lemmas.

LEMMA 1. $-1-34464\left(=h_{1}\right) \in\langle 6\rangle$.

LEMMA 2. $-4-6-232464\left(=h_{2}\right) \in(6)$.

LEMMA 3. $-47-34464 \quad\left(=h_{3}\right) \in\langle 6\rangle$.

LEMMA 4. $5 \in\langle 6\rangle$.

Hence $F(2,7)$ is cyclic, and the result follows from abelianizing the defining relations.

In the proofs of the lemmas underlined subwords in one line are replaced by equivalents in the next. Where this is not by direct application of one defining relation or by free reduction the annotations describe how the equivalent is calculated. A dot in a word indicates that a subword with freely trivial value will be inserted in the corresponding position in the following line. 
Proof of Lemma 1. We prove $-1-34464=6^{10}$ by showing $-4316^{10}-4-6-4=e:$

$$
\begin{aligned}
& -431.66 \underline{6} .66 \underline{6} .66 \underline{6} \cdot 6-4-6-4 \\
& =-4312-24-4661 \cdot 1-24-4661 \cdot 1-24-4661 \cdot 1-24-46-4-6-4 \\
& =-433-2423-31-2423-31-2423-3 \cdot 1-245-4-6-4 \\
& =-43 \cdot 35-2 \cdot 5-2 \cdot 5-35-5-44-556-61 \cdot 33-6-4 \\
& =-4 \underline{3-2235} \cdot \underline{-27}-\underline{-75} \cdot-27-754-6-3771-133 \cdot-6-4 \\
& =-41.67-72-2-1-61-1-77-1-5-6 \cdot-3724-55-6-4 \\
& =-41-7711 \cdot-3 \cdot 7 \cdot-3-22-3 \cdot-6-4-4 \\
& =5212-2-35-57-55-4-1-77-6-4-4 \\
& =5 \underline{4-244} \underline{5-4-2-2} \\
& =56-7 \\
& =e \text {; }
\end{aligned}
$$

(1) $6=1-7=11-2$

(2) $\underline{-4661}=\underline{561}=71=2$

(3) $\underline{-2423}=3 \underline{23}=34=5$

(5) $\underline{235}=45=6, \underline{-754}=-64=-5, \underline{-133}=23=4$

(6) $-1-77-1-5-6=-2 \underline{-6-5}-6=-\underline{-1}-\underline{-6}=-2-1=-3,72 \underline{4-5}=7 \underline{2}-\underline{3}=7-1=-6$

(7) $-\underline{-1-7}=-46=5$

(8) $212=23=4, \underline{-57}-5=6-5=4, \underline{7-6}-4-4=\underline{5-4}-4=3-4=-2$

(9) $4-244=4 \underline{34}=45=6, \underline{5-4}-2-2=\underline{3-2-2}=1-2=-7$

(10) defining relation 5 .

Proof of Lemma 2. Let $x=6^{2} h_{1}^{-1} 6^{-2} h_{1} 6^{2}\left(=6^{2}\right)$. We prove $-4-6-232464=6^{-35}$ by showing $2464\left(6^{4} h_{1}-6 x h_{1} 6^{-2} x h_{1}\right)-4-6-23=e$ :

$$
\begin{aligned}
& 24646666 h_{1}-6 x h_{1}-6 \cdot-6 x h_{1}-4-6-23 \\
& =24646666 h_{1} 7-1 x-1-344-2264-62-27-1 x-1-344-23 . \\
& \text { Consider }-1 \underline{x}-1-344-2 \\
& =-16 \cdot 6-4-6-4-431-6-6-1 \cdot-3446466-1-344-2 \\
& =1-24-46-4 \cdot-6-4-4343-274-4-3446.4-3-4-344 .-2 \\
& =133-445-53-3-77-6-4-4 \cdot 51723-5.446-77-13-5 \cdot 44-55 \cdot-2 \\
& =116-4-2-2-27-751723-57-72-63-57-725-445-53-3-2 \\
& =116-4-2-3772 \cdot \underline{361}-6 \cdot \underline{361} \cdot 14-31-7-4-4 \\
& =116-4 \underline{33-4} 4551-6 .-4 \underline{451-7714}-31-7-4-4
\end{aligned}
$$


$=116-41651-6-55-434-31-7-4-4$

$=.1 .1 \underline{65235}-2-7-4-4$

$=6-61-66-556-611-2-7-4-4$

$=63$.

Substitute (13) for (3) in (2)

$(1)=2464 \cdot \underline{66} \underline{6} \underline{h_{1}} \cdot 76326 \underline{4-62}-1633$

$=246.4-3347-1-72-3556-1-3446.45-576326 .-3-3-1633$

$=246-77-1-2 \cdot-7-1-5 \cdot 446-771-166.632 \cdot 6-55-44-3-3-1633$

$=-1-6-\underline{2}-11-7-1-57-722-7 \cdot 6-55632-334 \underline{3-1-16} \cdot 33$

$=-1-6-36-73-72-2473 \cdot-1 \cdot \underline{51-6-5563} \cdot 3$

$=.-7373-22-1-66773-223$

$=-66-7325124$

$=-645$

$=e$;

(1) $-6=7-1, h_{1}=-1-344.64=-1-344-2264$,

$h_{1}-4-6=-1-34 \underline{64-.4-6}=-1-344$

(4) $-16=-7=1-2,1-6 \underline{-6}=1-\underline{6-5}-\underline{4}=\underline{1-7}-53=\underline{6-53}=43$, similarly $66-1=-3-4,-1=-27$

(5) $\underline{-46-4}=5-4=3, \underline{4-4-3}=23-5,4-3=2=-13$

(6) $3 \underline{3-4}=3-2=1, \underline{-3}-7=-2-1-7=-2-2, \underline{7-6}-4-4=\underline{5-4}-4=3-4=-2$, similarly $446-7=2,4 \underline{4-5}=4-3=2$

(7) $-2-27=-2-1=-3, \underline{-751}=-61=7, \underline{5-4} \cdot \underline{45}=\underline{3-223-31} \underline{-16}=14-31-7$

(8) $-2-37 \underline{7} \underline{2}=-2-37-6 \underline{1-1} 3=-2-3 \underline{7-63}=-2 \underline{-353}=\underline{-24} 3=33$, $\underline{36}=5 \underline{-445}=55$

(9) $3 \underline{3-4}=3-2=1, \underline{45} 51-771=6 \underline{6671}=\underline{67} \underline{71}=12=3$

(10) $-41651 \underline{-6-5}=-4165 \underline{1-7}=-4.1656=\underline{-46-6167}=511=52$

(11) $65 \underline{235}=65 \underline{45}=6 \underline{56}=67=1$

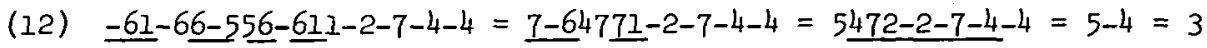

(14) $\underline{6} 6=\underline{456}=47,6=.45=\underline{-22}-3 \underline{34} 5=-1-72-355, \underline{4-62}=\underline{4-5}-\underline{42}=-3-3$

(15) $\underline{4-3} \underline{\underline{3}} \underline{\underline{z}-1}=\underline{2} \underline{\underline{5}-6}=-1 \underline{\underline{3}-4}=-1-2,5 \underline{6}-1-3=5 \underline{1-1}-3=\underline{5-6}-3=-4-3=-5$

(16) $24 \underline{6-7}=2 \underline{4-5}=2-3=-1,4 \underline{6-7}=4 \underline{4-5}=4-3=2, \underline{4-3}-3=2-3=-1$

(17) $\underline{1-7}-1-57=6-16=6-7, \underline{-722}=12=3,3-1-16=3-1-7=3-2=1$

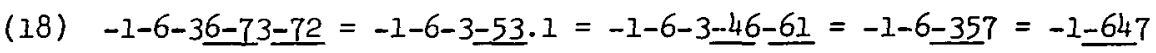
$=-1 \underline{-57}=-16=-7,51 \underline{-6-5}=\underline{11-7}=56=7$

(19) $7 \underline{3-2}=71=2, \underline{2-1}-6=7-6=5$ 
(20) $\underline{6-7} 325=\underline{-5325}=\underline{-425}=-35=4, \underline{124}=34=5$

(21) defining relation 4 .

Proof of Lemma 3 . We prove $-47-34464=6^{-35}$ by showing $-34464 h_{2}^{-1}-47=e$;

$$
\begin{aligned}
-34464 h_{2}^{-1}-47 \\
=-34 \cdot \underline{464-4-6-4-2-32.64-47} \\
=-3 \underline{45-53-3-2-32-11-7767} \\
=-3 \underline{6-4-5762} \\
=-312 \\
=e ;
\end{aligned}
$$

(3) $-53-\underline{3-2}-3=\underline{-53}-\underline{-4}=-4-5,7 \underline{67}=71=2$

(4) $6-\underline{-4}-576=\underline{-466}=6 \underline{56}=67=1$

(5) defining relation 1 .

Proof of Lemma 4. Let $y=h_{1} h_{3}^{-1}$. We prove $5=6^{255}$ by showing $-5\left(-6 y^{2} h y^{-2} 6 y^{2} 6 y^{2}-6 y^{-1}-6 y^{2} h y^{-2} 6 y^{2}-6 y^{-1}-6 y^{2} h y^{-2} 6 y^{2} 6 y^{2}\right)=e$.

$-5-6 \underline{y} \underline{y} h_{2}-\underline{y}-\underline{y} 6 \underline{y} \underline{6} 6 \underline{y} \underline{y}-6-\underline{y}-6 y \underline{y} h_{2}-y-y 6 y \underline{y}-6-y-6 y \underline{y} h_{2}-y-y 6 y \underline{y} 6 y \underline{y}$

$=-5-63-24 h_{2}-4 \underline{2-363}-246 \underline{3-24}-6-3-63-24 h_{2}-4 \underline{2}-363-24-6-3-6 \underline{3}-24 h_{2}-4 \underline{2}-363$ $-2463-24$

$=\underline{-5-61-6}-232 \underline{46-163}-24.614-\underline{6-3-61-6}-232 \underline{46-163}-24-\underline{6-3-61-6}-232 \underline{46-163}$

$=-2 \underline{32-24}-55614-5-2 \underline{32-24-5}-2 \underline{32-24-5} 5614$

$=-271 \underline{4-5}-2-2714$

$=-3-24$

$=e$;

(1) $y=h_{1} h_{3}^{-1}=-1-34464-4-6-4-43-74=-1-74=-24=3,-y=-3=-42$

(2) $4 h_{2}-4=\underline{4-4}-6-2324 \underline{4-4}=-6-23246$

(3) $-5 \underline{-61}-6=-5 \underline{I-6}=-55=e, 46 \underline{-16} 3=4 \underline{6-73}=\underline{4-53}=-33=e$,

$$
-6-\underline{-61}-6=-6-3 \underline{\underline{I}-6}=-6-\underline{-35}=-64=-5
$$

(4) $3 \underline{2}-24-5=\underline{3} 4-5=5-5=e$

(5) $-271=-22=e$ 
(6) defining relation 2 .

\section{References}

[1] A.M. Brunner, "The determination of Fibonacci groups", Buz2. Austral. Math. Soc. 11 (1974), 11-14.

[2] John J. Cannon, Lucien A. Dimino, George Havas and Jane M. Watson, "Implementation and analysis of the Todd-Coxeter algorithm", Math. Comp. 27 (1973), 463-490.

[3] J.H. Conway, "Advanced problem 5327", Amer. Math. Monthly 72 (1965), 915 .

[4] D.L. Johnson, J.W. Wamsley, and D. Wright, "The Fibonacci groups", Proc. London Math. Soc. (3) 29 (1974), 577-592.

[5] John Leech, "Coset enumeration", Computational problems in abstract algebra, 21-35 (Proc. Conf. Oxford, 1967. Pergamon, Oxford, London, Edịnburgh, New Yorh, Ioronto, Sydney, Paris, Brausschweig, 1970).

[6] John Leech, "Computer proof of relations in groups", Topics in group theory and computation (Proc. Royal Irish Acad. Summer School on Group Theory aü Computation, Galway 1973. Acaajemic Press, to appeur).

[7] Various Authors, "Generators and relations for cyclic groups" (Solution to advanced problem 5327, see [3] above), Amer. Math. Monthly 74 (1967), 91-93.

\footnotetext{
Department of Mathematics, Institute of Advanced Studies, Australian National University, Canberra, ACT.
} 